\title{
MASSIVE BLACK HOLES IN STELLAR SYSTEMS: “QUIESCENT” ACCRETION AND LUMINOSITY
}

\author{
M. Volonteri ${ }^{1}$, M. DotTI $^{2}$, D. CAMPBell ${ }^{1}$, And M. Mateo $^{1}$ \\ ${ }^{1}$ Astronomy Department, University of Michigan, Ann Arbor, MI 48109, USA \\ 2 Max Planck Institute for Astrophysics, Karl-Schwarzschild-Str. 1, 85741 Garching, Germany \\ Received 2010 November 3; accepted 2011 February 8; published 2011 March 15
}

\begin{abstract}
Only a small fraction of local galaxies harbor an accreting black hole, classified as an active galactic nucleus. However, many stellar systems are plausibly expected to host black holes, from globular clusters to nuclear star clusters, to massive galaxies. The mere presence of stars in the vicinity of a black hole provides a source of fuel via mass loss of evolved stars. In this paper, we assess the expected luminosities of black holes embedded in stellar systems of different sizes and properties, spanning a large range of masses. We model the distribution of stars and derive the amount of gas available to a central black hole through a geometrical model. We estimate the luminosity of the black holes under simple, but physically grounded, assumptions on the accretion flow. Finally, we discuss the detectability of "quiescent" black holes in the local universe.
\end{abstract}

Key words: black hole physics - galaxies: nuclei - galaxies: structure - stars: mass-loss

Online-only material: color figures

\section{INTRODUCTION}

Dynamical evidence indicates that massive black holes (MBHs) with masses in the range $M_{\mathrm{BH}} \sim 10^{6}-10^{9} M_{\odot}$ ordinarily dwell in the centers of most nearby galaxies (Ferrarese $\&$ Ford 2005). The evidence is particularly compelling in the case of our Galaxy, hosting a central black hole $(\mathrm{BH})$ with mass $\simeq 4 \times 10^{6} M_{\odot}$ (e.g., Schödel et al. 2003; Ghez et al. 2005). MBHs with smaller masses exist as well. For example, the Seyfert galaxies, POX 52 and NGC 4395, are thought to contain MBHs with mass $\sim 10^{5} M_{\odot}$ (Barth et al. 2004; Peterson et al. 2005). Low-mass BHs might also exist in dwarf galaxies, for instance in Milky Way satellites. If these BHs exist, they can help us understand the process that formed the seeds of the massive holes we detect in much larger galaxies (van Wassenhove et al. 2010). BHs in massive galaxies have a high probability that the central BH is not "pristine," that is, it has increased its mass by accretion or mergers. Dwarf galaxies undergo instead a quieter merger history, and as a result, if they host BHs, they still retain some "memory" of the original seed mass distribution (Volonteri et al. 2008).

The dynamical-mass estimates indicate that, across a wide range, central $\mathrm{BH}$ mass is about $0.1 \%$ of the spheroidal component of the host galaxy, with a possible mild dependence on mass (Magorrian et al. 1998; Marconi \& Hunt 2003; Häring \& Rix 2004). A tight correlation is also observed between the MBH mass and the stellar velocity dispersion of the hot stellar component (" $M-\sigma$ "; Ferrarese \& Merritt 2000; Gebhardt et al. 2000; Tremaine et al. 2002; Graham 2008; Gültekin et al. 2009b). Lauer et al. (2007) suggest that at least some of these correlations break down at the largest galaxy and BH masses (but see Bernardi et al. 2007; Tundo et al. 2007; Graham 2008). One unanswered question is whether this symbiosis extends down to the lowest galaxy and BH masses (Greene et al. 2008), due to changes in the accretion properties (Mathur \& Grupe 2005), dynamical effects (Volonteri 2007), or a cosmic bias (Volonteri \& Natarajan 2009; van Wassenhove et al. 2010).

It has also been proposed (e.g., Portegies Zwart et al. 2004; Gürkan et al. 2004) that BHs of intermediate mass (between the stellar mass range, $\sim$ few tens $M_{\odot}$, and the supermassive $\mathrm{BH}$ range, $\gtrsim 10^{5} M_{\odot}$ ), can form in the center of dense young star clusters. It is proposed that the formation of the $\mathrm{BH}$ is fostered by the tendency of the most massive stars to concentrate into the cluster core through mass segregation. The merging of mainsequence stars via direct physical collisions can enter into a runaway phase, forming a very massive star, which can then collapse to form a BH (Begelman \& Rees 1978; Ebisuzaki et al. 2001; Miller \& Hamilton 2002; Portegies Zwart \& McMillan 2002; Portegies Zwart et al. 2004; Freitag et al. 2006a, 2006b; Gürkan et al. 2004, 2006). Observational evidences for intermediate-mass BHs in globular clusters are scant (e.g., van der Marel \& Anderson 2010; Pasquato 2010, and references therein). Dynamical measurements are hampered by the small size of the sphere of influence of these $\mathrm{BHs}$, and only four candidates have currently been identified, in M15, M54, G1, and $\omega$ Centauri (Gerssen et al. 2002; Ibata et al. 2009; Gebhardt et al. 2005; Noyola et al. 2008). The radio and X-ray emission detected from G1 make this cluster the strongest candidate, although alternative explanations, such as an X-ray binary is possible (Ulvestad et al. 2007; Pooley \& Rappaport 2006).

"Massive" black holes (more massive than stellar mass black holes) are therefore expected to be widespread in stellar systems, from those of the lowest to highest mass. Only a small fraction of these MBHs are active at levels that are expected for active galactic nuclei (AGNs), and, indeed, most MBHs at the present day are "quiescent." However, because MBHs are embedded in stellar systems, they are unlikely to ever become completely inactive. An MBH surrounded by stars could be accreting material, either stripped from a companion star or available as recycled material via mass loss of evolved stars (Ciotti \& Ostriker 1997). Quataert (2004) models the gas supply in the central parsec of the Galactic center due to the latter process. Winds from massive stars can provide $\sim 10^{-3} M_{\odot} \mathrm{yr}^{-1}$ of gas, with a few percent, $\sim 10^{-5} M_{\odot} \mathrm{yr}^{-1}$, of the gas flowing in toward the central MBH. Quataert (2004) shows that the observed luminosity from Sgr A* can indeed be explained by relatively inefficient accretion of gas originating from stellar winds.

Elliptical galaxies with quiescent MBHs, systems for which we have both accurate MBH masses and data about their surroundings, hint that stellar winds may be a significant source 
of fuel for the MBH. The hot gas of the interstellar medium, lending itself to X-ray observations, cannot be the sole source of fuel for at least some MBHs. In particular, some MBHs are brighter than one would expect for inefficient accretion, but significantly less bright than for normal accretion (Soria et al. 2006a). The X-ray luminosity can vary by $\sim 3$ orders of magnitude displaying no relationship between MBH mass and the Bondi accretion rate (Pellegrini 2005). It is likely that warm gas that has not yet been thermalized or virialized originating from stellar winds and supernovae from near the MBH provides a significant amount of material for accretion, possibly an order of magnitude larger than the Bondi accretion rate of hot interstellar medium gas alone (Soria et al. 2006b).

We attempt in this paper a simple estimate of how much recycled gas is available for accretion onto an $\mathrm{MBH}$ in different stellar systems, from globular clusters to galaxies, including dwarf spheroidals, nuclear star clusters in the cores of latetype galaxies and early-type normal galaxies. We show that the amount of fuel available to MBHs through stellar winds in quiescent galaxies is indeed meager, and unless extreme conditions are met, X-ray detection of MBHs in globular clusters and low-mass galaxies is expected to be uncommon.

\section{METHOD}

\subsection{Stellar Models}

To model the accretion rate, we must choose threedimensional stellar distributions for the various stellar systems we consider here. For globular clusters and dwarf spheroidals, we assume the stars to be distributed following a Plummer profile:

$$
\rho(r)=\frac{3}{4 \pi} \frac{M_{\text {stellar }}}{a^{3}}\left(1+\frac{r^{2}}{a^{2}}\right)^{-5 / 2},
$$

where $a=R_{\text {eff }}$ is the core radius.

Early-type galaxies and nuclear clusters are modeled as Hernquist spheres:

$$
\rho(r)=\frac{M_{\text {stellar }}}{2 \pi} \frac{r_{h}}{r\left(r+r_{h}\right)^{3}},
$$

where the scale length $r_{h} \approx R_{\text {eff }} / 1.81$. To fully define the stellar systems we have only to relate the stellar mass, $M_{\text {stellar }}$, to the effective radius, $R_{\text {eff }}$.

For globular clusters, we recall that simulations by Baumgardt et al. $(2004$, 2005) suggest that globular clusters with MBHs have relatively large cores $a \sim 1-3$ pc (see also Trenti et al. 2007). Consistent results were found using Monte Carlo simulations (Umbreit et al. 2009) and in analytical models (Heggie et al. 2007). The core radii (where measured) of globular clusters, hosting intermediate-mass BH candidates, are roughly consistent with the values we considered, ranging from approximately $0.5 \mathrm{pc}$ in M15 (Gerssen et al. 2002; core radius from the catalog presented in Harris et al. $2010^{3}$ ), up to few pc in $\omega$ Centauri (Noyola et al. 2008).

For early-type galaxies, we adopt the fits by Shen et al. (2003) for stellar mass versus effective radius in Sloan Digital Sky Survey galaxies:

$$
R_{\text {eff }}=2.5\left(\frac{M_{\text {stellar }}}{4 \times 10^{10} M_{\odot}}\right)^{0.56} \mathrm{kpc} .
$$

\footnotetext{
3 http://physwww.physics.mcmaster.ca/harris/mwgc.dat
}

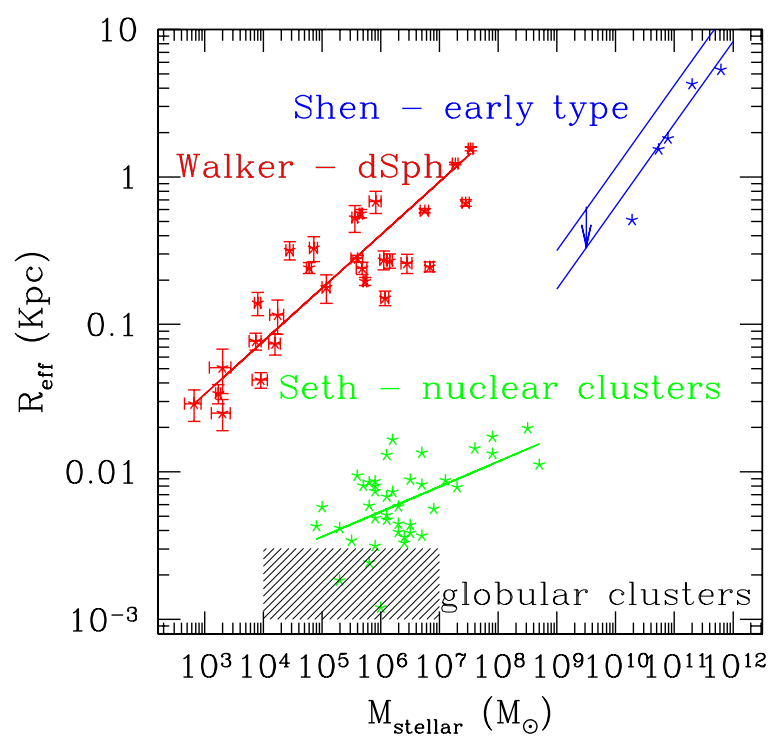

Figure 1. Relationship between half-mass radii and stellar mass for different galaxy morphological types. For dwarf spheroidals and nuclear clusters we show the data along with our best fit. For elliptical galaxies we show the effective radii of five galaxies from Soria et al. (2006a), along with Shen et al. (2003) fit and a correction of a factor 0.55 . We include as a shaded area the range in half-mass radii and stellar mass adopted for globular clusters.

(A color version of this figure is available in the online journal.)

The scatter is roughly $0.2 \mathrm{dex}$ for stellar masses between $10^{8} M_{\odot}$ and $10^{10} M_{\odot}: \sigma_{\ln R}=0.34+0.13 /\left[1+\left(M_{\text {stellar }} / 4 \times 10^{10} M_{\odot}\right)\right]$.

We note that for five galaxies (NGC 4697, NGC 3377, NGC 4564, NGC 5845, NGC 821) where measurements of the effective radius are available (along with stellar masses, $\mathrm{BH}$ masses, and gas density - see Soria et al. 2006a and Marconi \& Hunt 2003) the fits derived by Shen et al. (2003) provide values of the effective radius roughly $55 \%$ times larger than the measured value. This is likely due to Shen et al. (2003) definition of effective radius as the radius enclosing $50 \%$ of the Petrosian flux. This definition differs from the standard definition of projected radius enclosing half of the total luminosity. We therefore scale the fit for early-type galaxies by a factor of 0.55 for consistency. As shown below (Figure 3), this small correction does not influence the accretion rate we derive.

For dwarf spheroidals, we fit the data presented in Walker et al. $(2009,2010)$. We assume a constant mass-to-light ratio of 2 for the visible component and derive stellar masses from the total luminosities:

$$
R_{\text {eff }}=0.93\left(\frac{M_{\text {stellar }}}{10^{7} M_{\odot}}\right)^{0.36} \mathrm{kpc},
$$

where the uncertainties in the slope and in the normalization are 0.06 and 0.2 dex, respectively. Finally, for nuclear clusters we fit the stellar mass versus effective radius data presented in Seth et al. (2008), leading to

$$
R_{\text {eff }}=7.9 \times 10^{-3}\left(\frac{M_{\text {stellar }}}{10^{7} M_{\odot}}\right)^{0.17} \mathrm{kpc},
$$

where the uncertainties in the slope and in the normalization are 0.05 and 0.3 dex, respectively. These scalings are shown in Figure 1. 


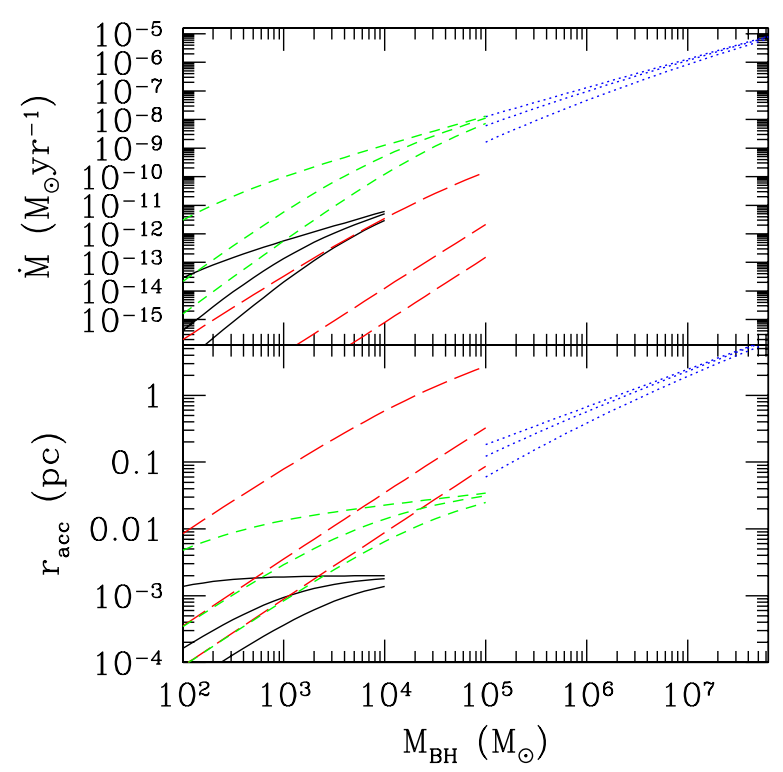

Figure 2. Top panel: accretion rate, in solar masses per year, onto a $\mathrm{BH}$ in a stellar system with $M_{\text {stellar }}=10^{3} M_{\mathrm{BH}}$. In each set of curves the wind velocity varies from $100 \mathrm{~km} \mathrm{~s}^{-1}$ (bottom) to $50 \mathrm{~km} \mathrm{~s}^{-1}$ (middle) to $10 \mathrm{~km} \mathrm{~s}^{-1}$ (top). Solid curves: globular clusters. Long-dashed curves: dwarf spheroidals. Shortdashed curves: nuclear star clusters. Dotted curves: early-type galaxies. Bottom panel: accretion radius for the same systems.

(A color version of this figure is available in the online journal.)

\subsection{Geometrical Model}

We develop here a simple geometrical model to estimate the accretion rate onto an $\mathrm{MBH}$ in a stellar system, fueled by mass loss from stars (Quataert et al. 1999). If a star is located at a distance $r$ from the $\mathrm{MBH}$, and if it produces an isotropic wind, with velocity $v_{\text {wind }}$, only the fraction of gas which passes within the accretion radius of the $\mathrm{MBH}$,

$$
R_{\mathrm{acc}}=2 G M_{\mathrm{BH}} /\left(v_{\mathrm{wind}}^{2}+\sigma^{2}+c_{s}^{2}\right),
$$

can be accreted (ignoring gravitational focusing). Here, $\sigma^{2}=$ $G M_{\text {stellar }} /\left(2.66 r_{h}\right)$ is the velocity dispersion of the stellar system at the half-mass radius. For a Hernquist profile, where the density in the inner region $\rho \propto r^{-1}$, the velocity dispersion decreases toward the center. Estimating $\sigma$ at the half-mass radius gives a conservative lower limit to the accretion radius, and hence the accretion rate. Following Miller \& Hamilton (2002), we assume that in Equation (6) the sound speed $c_{s}=10 \mathrm{~km} \mathrm{~s}^{-1}$, and, $v_{\text {wind }}=50 \mathrm{~km} \mathrm{~s}^{-1}$ as reference values, although we study the effect that a different $v_{\text {wind }}$ has on our model (see Figure 2).

If $\sigma \gg v_{\text {wind }}, R_{\text {acc }}$ depends only on the properties of the potential well of the stellar distribution, not on the wind properties. In particular, $R_{\mathrm{acc}} \simeq M_{\mathrm{BH}} R_{\mathrm{eff}} / M_{\text {stellar }} \simeq 10^{-3} R_{\mathrm{eff}}$ if $M_{\text {stellar }}=10^{3} M_{\mathrm{BH}}$. Note that, at fixed BH mass, the more massive the galaxy, the smaller $R_{\text {acc }}$ is, as the scaling of $R_{\text {eff }}$ with $M_{\text {stellar }}$ is a power law with exponent less than one (see, e.g., Equation (3)). On the other hand, if $\sigma \ll v_{\text {wind }}, R_{\text {acc }}$ depends only on the wind velocity. These two limits are apparent in Figures 2 and 3, and they will be discussed in the next section.

Geometrical considerations suggest that for $r>R_{\text {acc }}$

$$
\dot{M}_{\mathrm{acc}, *}=\frac{1}{2} \dot{M}_{*}\left[1-\left(1-\frac{R_{\mathrm{acc}}^{2}}{r^{2}}\right)^{1 / 2}\right],
$$

where $\dot{M}_{*}$ is the mass-loss rate from the star. If the star lies within $R_{\text {acc }}$, we consider $\dot{M}_{\text {acc, } *}=\dot{M}_{*}$. Equation (7) implicitly

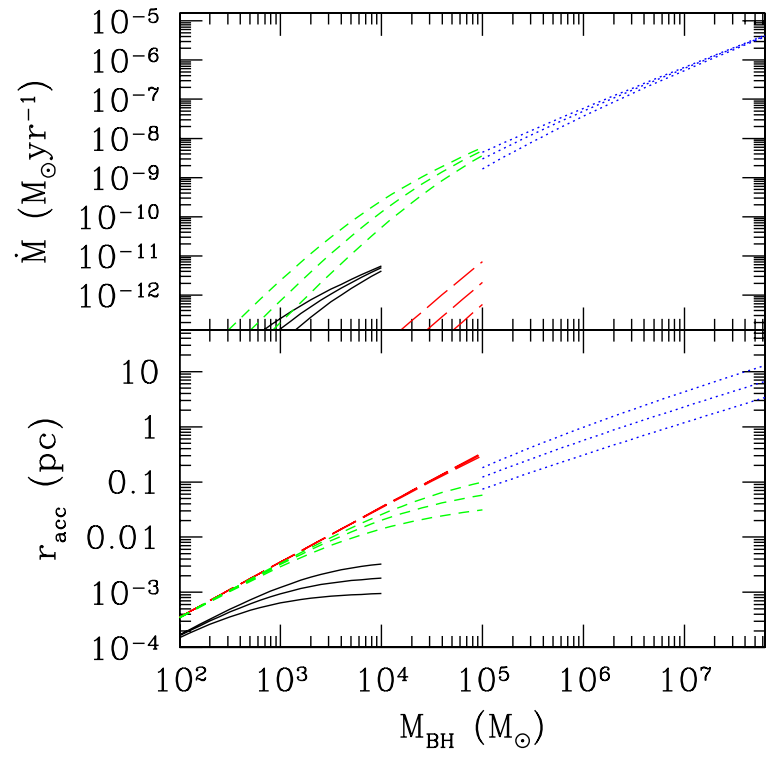

Figure 3. Top panel: accretion rate, in solar masses per year, onto a $\mathrm{BH}$ in a stellar system with $M_{\text {stellar }}=10^{3} M_{\mathrm{BH}}$. In each set of curves we vary the size of the stellar system. For globular clusters we assume $R_{\mathrm{eff}}=1 \mathrm{pc}$ (top); $R_{\mathrm{eff}}=2 \mathrm{pc}$ (middle); $R_{\mathrm{eff}}=4 \mathrm{pc}$ (bottom). For galaxies, the middle curve shows the best-fit $R_{\text {eff }}$ at a given stellar mass (Equations (4), (5), and (6)), the top curves assume that $R_{\text {eff }}$ is half the best-fit value, and the bottom curves assume that $R_{\text {eff }}$ is twice the best-fit value. The wind velocity is fixed at $50 \mathrm{~km} \mathrm{~s}^{-1}$. Solid curves: globular clusters. Long-dashed curves: dwarf spheroidals. Short-dashed curves: nuclear star clusters. Dotted curves: early-type galaxies. Bottom panel: accretion radius for the same systems.

(A color version of this figure is available in the online journal.)

assumes that the stars have a spherically symmetric distribution and that their velocity field (and, as a consequence, the velocity field of the wind) is isotropic. In a rotating stellar system, the presence of net angular momentum of the gas can change the accretion rate onto the $\mathrm{BH}$ (e.g., Cuadra et al. 2008). A study of the dependence of the accretion rate on the degree of rotational support of the stellar distribution is beyond the scope of this paper.

The total contribution from all stars is found by integrating over the density profile of the stellar system:

$$
\dot{M}_{\mathrm{acc}}=\int_{R_{\mathrm{acc}}}^{\infty} 4 \pi r^{2} \frac{\rho(r)}{\left\langle m_{*}\right\rangle} \dot{M}_{\mathrm{acc}, *} d r
$$

where $\left\langle m_{*}\right\rangle$ is the mean stellar mass and $\rho$ is given by Equations (1) and (2). The normalization in Equation (8) is given by the cumulative mass-loss rate of all the stars in the stellar structure that we estimate following Ciotti et al. (1991):

$$
\dot{M}_{\mathrm{gal}}=1.5 \times 10^{-11} M_{\odot} \mathrm{yr}^{-1} \frac{L_{B}}{L_{B, \odot}}\left(\frac{t_{*}}{15 \mathrm{Gyr}}\right)^{-1.3}
$$

where $t_{*}$ is the age of the stellar population and $L_{B}$ is the total luminosity of the stellar system. We set $t_{*}=5 \mathrm{Gyr}$ for dSphs and nuclear star clusters, and $t_{*}=12 \mathrm{Gyr}$ for early-type galaxies and globular clusters. We derive $B$-band luminosities from stellar masses assuming a mass-to-light ratio of 5 in the $B$ band.

We obtain an upper limit of the luminosity of the MBH by assuming that the whole $\dot{M}_{\text {acc }}$ is indeed accreted by the MBH.

\subsection{Accretion Rate and Luminosity}

Figure 2 shows the resulting accretion rate for a central $\mathrm{MBH}$ in different stellar systems, where we assume that the MBH mass 


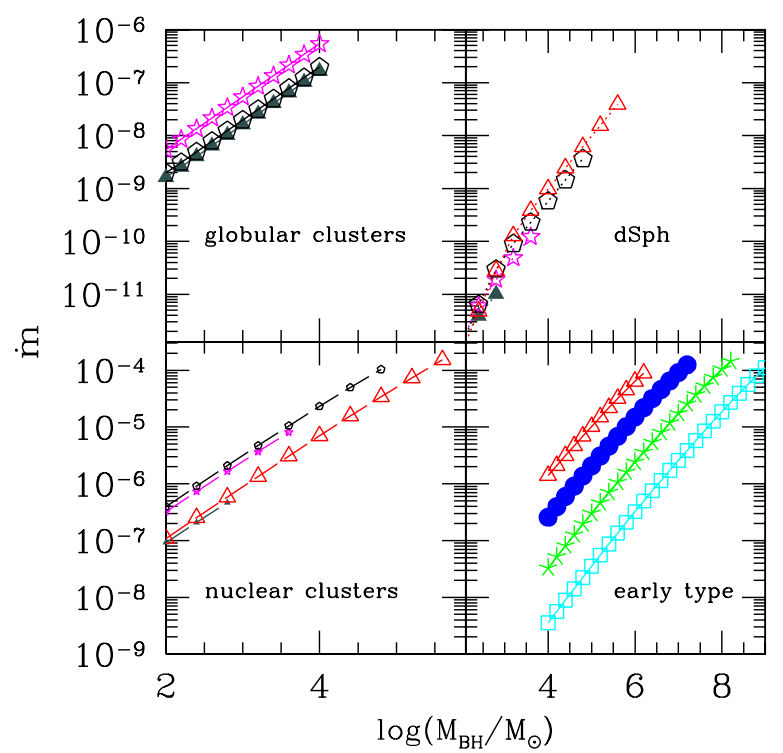

Figure 4. Accretion rate, in Eddington units, of MBHs in different stellar systems. Top right: dwarf spheroidals; bottom right: early-type galaxies; bottom left: nuclear clusters; top left: globular clusters. Gray filled triangles: $M_{\text {stellar }}=$ $10^{5} M_{\odot}$; magenta stars: $M_{\text {stellar }}=10^{6} M_{\odot}$; black pentagons: $M_{\text {stellar }}=10^{7} M_{\odot}$; red empty triangles: $M_{\text {stellar }}=10^{8} M_{\odot}$; blue dots: $M_{\text {stellar }}=10^{9} M_{\odot}$; green asterisks: $M_{\text {stellar }}=10^{10} M_{\odot}$; cyan squares: $M_{\text {stellar }}=10^{11} M_{\odot}$. The mass-size relationship is given by Equations (4), (5), and (6). We assume $R_{\mathrm{eff}}=2$ for globular clusters. The wind velocity is $v_{\text {wind }}=50 \mathrm{~km} \mathrm{~s}^{-1}$.

(A color version of this figure is available in the online journal.)

scales with the mass of stellar component, $M_{\mathrm{BH}}=10^{-3} M_{\text {stellar }}$ (Marconi \& Hunt 2003; Häring \& Rix 2004), and we have considered $v_{\text {wind }}$ a free parameter. We have assumed that $R_{\text {eff }}$ scales exactly with $M_{\text {stellar }}$ following the relationships discussed above. Note that for high values of the stellar masses in earlytype galaxies and nuclear star clusters, the accretion rate and $R_{\text {acc }}$ do not depend on the wind velocities. In these cases $\sigma \gg v_{\text {wind }}$, and the accretion rate depends only on the properties of the host stellar structure and on the BH mass (see the discussion of Equation (6) above).

In Figure 3 we instead fix $v_{\text {wind }}$ and allow for a scatter in the mass-size relationship. For globular clusters we assume $R_{\text {eff }}=1 \mathrm{pc}, R_{\text {eff }}=2 \mathrm{pc}$, and $R_{\text {eff }}=4 \mathrm{pc}$. For galaxies, the middle curve shows the best-fit $R_{\mathrm{eff}}$ for a given stellar mass value (Equations (1), (2), and (3)), the top curves assume that $R_{\text {eff }}$ is half the best-fit value, and the bottom curves assume that $R_{\text {eff }}$ is twice the best-fit value. We have assumed $M_{\text {stellar }}=$ $10^{5}-10^{7} M_{\odot}$ for globular clusters, $M_{\text {stellar }}=10^{5}-10^{8} M_{\odot}$ for dwarf spheroidals and nuclear star clusters, and $M_{\text {stellar }}=$ $10^{8}-10^{11} M_{\odot}$ for early-type galaxies, limiting our investigation to the mass ranges probed by Shen et al. (2003), Walker et al. (2009), and Seth et al. (2008). In this plot the $v_{\text {wind }} \gg \sigma$ limit of Equation (6) becomes evident: at low stellar masses, for every type of stellar distribution but for the early-type galaxies, $R_{\mathrm{acc}}$ does not depend on $R_{\text {eff }}$, and it is determined only by the $\mathrm{BH}$ mass and the assumed $v_{\text {wind }}$. The early-type galaxies generate deeper potential wells, never reaching the $v_{\text {wind }} \gg \sigma$ limit.

The bolometric luminosity of the $\mathrm{MBH}$ can be written as

$$
L_{\mathrm{bol}}=\epsilon \dot{M} c^{2} \text {, }
$$

where $\epsilon$ represents the fraction of the accreted mass that is radiated away. The nature of the accretion process, and the consequent value of $\epsilon$, is rather uncertain. AGNs accrete through accretion disks with a high efficiency $(\epsilon \sim 0.1)$. Supermassive

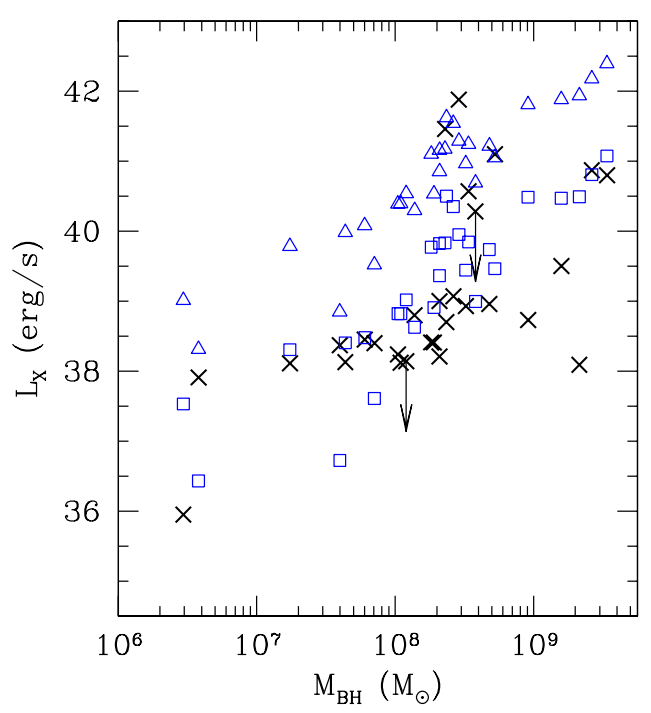

Figure 5. X-ray luminosity (top) of MBHs in 29 nearby elliptical galaxies. Crosses and upper limits are from Pellegrini (2010), where we select only BHs with dynamical mass measurement. Triangles: "radiatively efficient" model. Squares: "radiatively inefficient" model.

(A color version of this figure is available in the online journal.)

BHs at the centers of quiescent galaxies, including the Milky Way, can have luminosities as low as $\sim 10^{-9}$ to $10^{-8}$ of their Eddington values (e.g., Loewenstein et al. 2001), and well below the luminosity one would estimate assuming $\epsilon \sim 0.1$.

Following Merloni \& Heinz (2008), we define $\lambda \equiv$ $L_{\text {bol }} / L_{\text {Edd }}$, where $L_{\text {bol }}$ is the bolometric luminosity and $L_{\mathrm{Edd}}=$ $4 \pi G M_{\mathrm{BH}} m_{\mathrm{p}} c / \sigma_{\mathrm{T}} \simeq 1.3 \times 10^{38}\left(M_{\mathrm{BH}} / M_{\odot}\right) \mathrm{erg} \mathrm{s}^{-1}$ is the Eddington luminosity. We write the radiative efficiency, $\epsilon$, as a combination of the accretion efficiency, $\eta$, that depends only on the location of the innermost stable circular orbit, ${ }^{4}$ here assumed to be $\eta=0.1$, and of a term, $\eta_{\text {acc }}$, that depends on the properties of the accretion flow itself: $\epsilon=\eta \eta_{\text {acc }}$. We also define $\dot{m}=\eta \dot{M} c^{2} / L_{\text {Edd }}$.

For "radiatively efficient" accretion, $\eta_{\text {acc }}=1$. To estimate the X-ray luminosity, we apply a simple bolometric correction and assume that the $\mathrm{X}$-ray luminosity is a fraction $\eta_{\mathrm{X}}$ of the bolometric luminosity. Ho et al. (1999) suggest that for low-luminosity AGN, with Eddington rates between $10^{-6}$ and $10^{-3}$ the luminosity on the $[0.5-10] \mathrm{keV}$ band represents a fraction $0.06-0.33$ of the bolometric luminosity. We assume here $\eta_{\mathrm{X}}=0.1$, so that $L_{\mathrm{X}}=\eta_{\mathrm{X}} \epsilon \dot{M} c^{2}$, where $\epsilon=\eta=0.1$. We refer to this model as "radiatively efficient."

Since the accretion rates we find are very sub-Eddington, we assume, in a second model, that the accretion flow is optically thin and geometrically thick. In this state the radiative power is strongly suppressed (e.g., Narayan \& Yi 1994; Abramowicz et al. 1988). Merloni \& Heinz (2008) suggest that this transition occurs at $\dot{m}<\dot{m}_{\mathrm{cr}}=3 \times 10^{-2}$, and that $\eta_{\text {acc }}=\left(\dot{m} / \dot{m}_{\mathrm{cr}}\right)$, so that $\epsilon=\eta\left(\dot{m} / \dot{m}_{\mathrm{cr}}\right)$. The X-ray luminosity is therefore: $L_{\mathrm{X}}=\eta_{\mathrm{X}} \epsilon \dot{M} c^{2}$, where again $\eta_{\mathrm{X}}=0.1$. We refer to this model as "radiatively inefficient."

In Figure 4 we show the accretion rate, in Eddington units, when we assume $\eta=0.1$. Hereafter, we vary the mass of the $\mathrm{MBH}$ from $100 M_{\odot}$ to $10^{4} M_{\odot}$ for globular clusters, since there is no firm conclusion that MBHs' masses scale with the mass of

\footnotetext{
4 If the viscous torque vanishes at the innermost stable circular orbit, then $\eta$ is a function of $\mathrm{BH}$ spin only, ranging from $\eta \simeq 0.057$ for Schwarzschild (non-spinning) $\mathrm{BHs}$ to $\eta \simeq 0.42$ for maximally rotating Kerr BHs.
} 

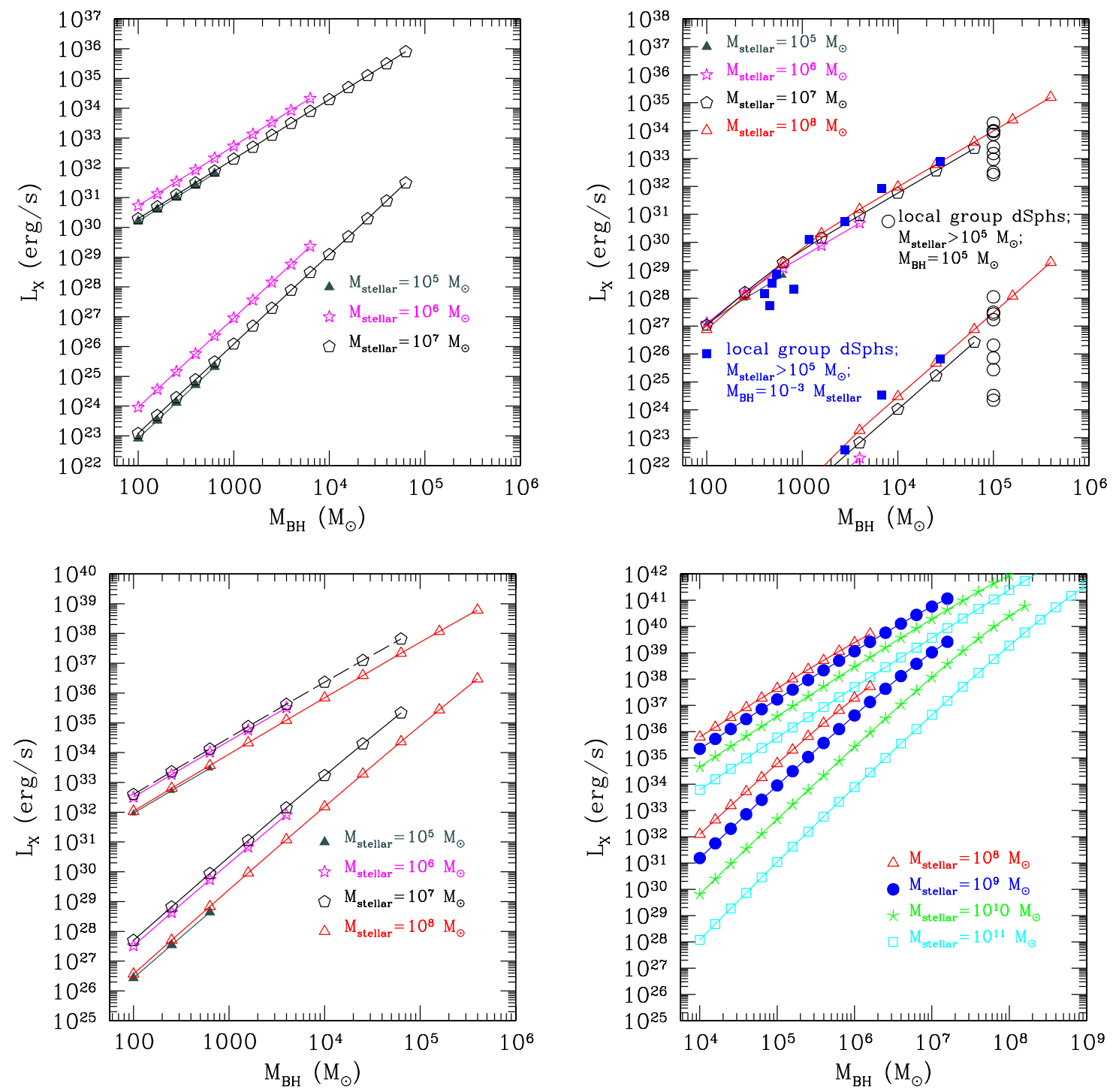

Figure 6. X-ray luminosity of MBHs in different stellar systems, assuming a radiatively efficient accretion flow (top series of curves) and a radiatively inefficient accretion flow (bottom series of curves). The wind velocity is $v_{\text {wind }}=50 \mathrm{~km} \mathrm{~s}^{-1}$. Top left: globular clusters. Top right: dwarf spheroidals. (Squares: luminosity we derive for dSphs with stellar mass $>10^{5} M_{\odot}$ assuming MBHs with mass $10^{-3}$ times the stellar mass, and using dynamical masses and radii from Walker et al. (2009). Open circles: luminosity an $M_{\mathrm{BH}}=10^{5} M_{\odot} \mathrm{MBH}$ would have in the same galaxies.) Bottom left: nuclear clusters. Bottom right: early-type galaxies. Gray filled triangles: $M_{\text {stellar }}=10^{5} M_{\odot}$; magenta stars: $M_{\text {stellar }}=10^{6} M_{\odot}$; black pentagons: $M_{\text {stellar }}=10^{7} M_{\odot}$; red empty triangles: $M_{\text {stellar }}=10^{8} M_{\odot}$; blue dots: $M_{\text {stellar }}=10^{9} M_{\odot}$; green asterisks: $M_{\text {stellar }}=10^{10} M_{\odot}$; cyan squares: $M_{\text {stellar }}=10^{11} M_{\odot}$. We have assumed $M_{\text {stellar }}=10^{5}-10^{7} M_{\odot}$ for globular clusters; $M_{\text {stellar }}=10^{5}-10^{8} M_{\odot}$ for dwarf spheroidals and nuclear star clusters, and $M_{\text {stellar }}=10^{8}-10^{11} M_{\odot}$ for early-type galaxies, limiting our investigation to the mass ranges probed by Shen et al., Walker et al., and Seth et al.

(A color version of this figure is available in the online journal.)

the stellar component as $M_{\mathrm{BH}}=10^{-3} M_{\text {stellar }}$. For galaxies we assume instead an upper limit to the MBH mass corresponding to $M_{\mathrm{BH}}=2 \times 10^{-2} M_{\text {stellar }}$, a lower limit of $100 M_{\odot}$ for $\mathrm{dSph}$ and nuclear clusters and a lower limit of $10^{4} M_{\odot}$ for early-type galaxies.

We complete the exercise by adding observational results for a sample of 29 early-type galaxies where both dynamical $\mathrm{BH}$ mass and X-ray luminosity (Pellegrini 2010) ${ }^{5}$ are available (see also Soria et al. 2006a; Gültekin et al. 2009a). Twenty-four of

\footnotetext{
5 The sample of Pellegrini (2010) comprises 112 galaxies with measured $\mathrm{X}$-ray luminosity. For systems that do not have dynamical $\mathrm{BH}$ mass measurement, Pellegrini (2010) derives BH masses from the $M-\sigma$ relation. We limit our analysis to those galaxies that have a direct $\mathrm{BH}$ mass measurement to avoid adding additional uncertainties, especially below $M_{\mathrm{BH}}=10^{7} M_{\odot}$, where the $M-\sigma$ relation is less secure. We note, however, that the results we discuss hold for the whole sample.
}

these galaxies also report the stellar mass of the bulge (Marconi $\&$ Hunt 2003). For those galaxies where the bulge mass is unavailable we derive stellar masses from $B$-band magnitudes. For these galaxies we also derive $B$-band luminosities directly from $L_{V}$ (Gültekin et al. 2009b), assuming $B-V=1$ (Coleman et al. 1980), and we check that our choice of a mass-to-light ratio of 5 agrees well with this complementary technique to derive $L_{B}$.

Figure 5 compares the luminosities we predict for these galaxies to the measured X-ray luminosity of the galaxies (or upper limits). In agreement with the conclusions of Pellegrini (2005) and Soria et al. (2006b) the radiatively inefficient case best fits the luminosity of most systems, except the most luminous ones. Overall, even the radiatively inefficient case slightly overestimates the luminosity, at least at the high-mass end, and we find that, for instance, $\eta_{\mathrm{X}}=0.03$ provides a much 
better fit. As discussed by Pellegrini (2010), there seems to be a smooth transition between radiatively inefficient and radiatively efficient accretion.

We also estimate the X-ray luminosities for Milky Way dSphs with stellar mass $>10^{5} M_{\odot}$, where we use directly $R_{\text {half }}$ and $M_{\text {stellar }}$ from Walker et al. (2010). We assume in one case that $M_{\mathrm{BH}}=10^{-3} M_{\text {stellar }}$, and in another case that BHs have a fixed MBH mass of $10^{5} M_{\odot}$, based on models presented in van Wassenhove et al. (2010). We note that in all these cases the X-ray luminosities for MBHs in dwarf galaxies are below $10^{35} \mathrm{erg} \mathrm{s}^{-1}$. Figure 6 summarizes our primary results: predicted $\mathrm{X}$-ray luminosities for different stellar systems.

\section{DISCUSSION}

We have developed a simple model to estimate the level of accretion fueled by recycled stellar winds on BHs hosted in stellar systems of different types. Let us examine the various assumptions of our models to question if our approach is too conservative. To model the accretion rate we need (1) a stellar density profile, (2) physical size and mass of a system, (3) a total mass loss from stars (which depends on their age and luminosity), and (4) a velocity of stellar wind.

Regarding points (3) and (4), we note that our choice of stellar ages and mass-to-light ratios are already quite optimistic (except for the case of globular clusters and early-type galaxies, but we note that our results for globulars are consistent with the estimate of Miller \& Hamilton 2002), and for most MBHs in massive stellar systems the wind velocity is not highly influential. Regarding point (2), we can see from Figure 3 that the relationship between size and radius does not have a very strong effect on our results. More interesting is point (1). As long as the wind velocity is larger than the velocity dispersion of a galaxy, the amount of available gas will increase if the density profile is steeper. For instance, an ideal density profile is an isothermal sphere (possibly singular) where the velocity dispersion is constant, while the central density increases toward the center. In such case the size of the accretion radius, and the accretion rate, is maximized (see Equations (6) and (7)).

One of our goals was to assess the detectability of putative MBHs in Milky Way dSphs. If they exist, they provide valuable information on the process that formed the seeds of the massive holes we detect in much larger galaxies (van Wassenhove et al. 2010). Figure 6 suggests that such BHs would be elusive, as the expected luminosities are often even less than those of X-ray binaries. Regarding the three points discussed above, in the case of dSphs, the observed stellar density profiles are very shallow and the central stellar densities are low, of order of at most a few stars per cubic parsec (Irwin \& Hatzidimitriou 1995). We therefore consider the choice of a steeper profile inappropriate. The analytical fit of the mass-size relationship (Equation (4)) could be inaccurate, but as we show in Figure 6, where we model specific galaxies using their measured masses and radii, we find that the luminosities are in very good agreement with what we find using the analytical fit. Finally, even assuming a wind velocity $v_{\text {wind }}=10 \mathrm{~km} \mathrm{~s}^{-1}$ the luminosities are always below $10^{38} \mathrm{erg} \mathrm{s}^{-1}$, even pushing the stellar age to $1 \mathrm{Gyr}$.

On the other hand, our model could indeed be too conservative for the case of nuclear star clusters. These systems have a wide spread in stellar ages (e.g., Carollo et al. 2001) and they exhibit steep density profiles (see Kormendy \& Kennicutt 2004 for a comprehensive review). Decreasing the typical stellar age to 1 Gyr increases the luminosity by about a factor 10 , while modeling the density profile as a singular isothermal sphere increases the luminosity by several orders of magnitude, with BHs with masses $>10^{4} M_{\odot}$ hosted in nuclear clusters with mass $>10^{7} M_{\odot}$ attaining luminosities $>10^{38} \mathrm{erg} \mathrm{s}^{-1}$. At luminosities below $>10^{38} \mathrm{erg} \mathrm{s}^{-1}$, the contamination from X-ray binaries is high (Gallo et al. 2010, and references therein) and we consider this to be a "safe" threshold for BH detection. Surveys built in the same spirit of AMUSE-Virgo and AMUSE-field, with a limiting luminosity of order $>10^{38} \mathrm{erg} \mathrm{s}^{-1}$, are likely to provide an excellent venue to test our models.

We thank E. Gallo and D. Maitra for fruitful discussions, and M. Walker for clarifications on his data.

\section{REFERENCES}

Abramowicz, M. A., Czerny, B., Lasota, J. P., \& Szuszkiewicz, E. 1988, ApJ, 332,646

Barth, A. J., Ho, L. C., Rutledge, R. E., \& Sargent, W. L. W. 2004, ApJ, 607, 90

Baumgardt, H., Makino, J., \& Hut, P. 2005, ApJ, 620, 238

Baumgardt, H., Portegies Zwart, S. F., McMillan, S. L. W., Makino, J., \& Ebisuzaki, T. 2004, in ASP Conf. Ser. 322, The Formation and Evolution of Massive Young Star Clusters, ed. H. J. G. L. M. Lamers, L. J. Smith, \& A. Nota (San Francisco, CA: ASP), 459

Begelman, M. C., \& Rees, M. J. 1978, MNRAS, 185, 847

Bernardi, M., Sheth, R. K., Tundo, E., \& Hyde, J. B. 2007, ApJ, 660, 267

Carollo, C. M., Stiavelli, M., de Zeeuw, P. T., Seigar, M., \& Dejonghe, H. 2001, ApJ, 546, 216

Ciotti, L., D’Ercole, A., Pellegrini, S., \& Renzini, A. 1991, ApJ, 376, 380

Ciotti, L., \& Ostriker, J. P. 1997, ApJ, 487, L105

Coleman, G. D., Wu, C., \& Weedman, D. W. 1980, ApJS, 43, 393

Cuadra, J., Nayakshin, S., \& Martins, F. 2008, MNRAS, 383, 458

Ebisuzaki, T., et al. 2001, ApJ, 562, L19

Ferrarese, L., \& Ford, H. 2005, Space Sci. Rev., 116, 523

Ferrarese, L., \& Merritt, D. 2000, ApJ, 539, L9

Freitag, M., Gürkan, M. A., \& Rasio, F. A. 2006a, MNRAS, 368, 141

Freitag, M., Rasio, F. A., \& Baumgardt, H. 2006b, MNRAS, 368, 121

Gallo, E., Treu, T., Marshall, P. J., Woo, J.-H., Leipski, C., \& Antonucci, R. 2010, ApJ, 714, 25

Gebhardt, K., Rich, R. M., \& Ho, L. C. 2005, ApJ, 634, 1093

Gebhardt, K., et al. 2000, ApJ, 539, L13

Gerssen, J., van der Marel, R. P., Gebhardt, K., Guhathakurta, P., Peterson, R. C., \& Pryor, C. 2002, AJ, 124, 3270

Ghez, A. M., Salim, S., Hornstein, S. D., Tanner, A., Lu, J. R., Morris, M., Becklin, E. E., \& Duchêne, G. 2005, ApJ, 620, 744

Graham, A. W. 2008, ApJ, 680, 143

Greene, J. E., Ho, L. C., \& Barth, A. J. 2008, ApJ, 688, 159

Gültekin, K., Cackett, E. M., Miller, J. M., Di Matteo, T., Markoff, S., \& Richstone, D. O. 2009a, ApJ, 706, 404

Gültekin, K., et al. 2009b, ApJ, 698, 198

Gürkan, M. A., Fregeau, J. M., \& Rasio, F. A. 2006, ApJ, 640, L39

Gürkan, M. A., Freitag, M., \& Rasio, F. A. 2004, ApJ, 604, 632

Häring, N., \& Rix, H.-W. 2004, ApJ, 604, L89

Harris, W. E. 2010, arXiv:1012.3224

Heggie, D. C., Hut, P., Mineshige, S., Makino, J., \& Baumgardt, H. 2007, PASJ, 59, L11

Ho, L. C. 1999, ApJ, 516, 672

Ibata, R., et al. 2009, ApJ, 699, L169

Irwin, M., \& Hatzidimitriou, D. 1995, MNRAS, 277, 1354

Kormendy, J., \& Kennicutt, R. C., Jr. 2004, ARA\&A, 42, 603

Lauer, T. R., et al. 2007, ApJ, 662, 808

Loewenstein, M., Mushotzky, R. F., Angelini, L., Arnaud, K. A., \& Quataert, E. 2001, ApJ, 555, L21

Magorrian, J., et al. 1998, AJ, 115, 2285

Marconi, A., \& Hunt, L. K. 2003, ApJ, 589, L21

Mathur, S., \& Grupe, D. 2005, ApJ, 633, 688

Merloni, A., \& Heinz, S. 2008, MNRAS, 388, 1011

Miller, M. C., \& Hamilton, D. P. 2002, MNRAS, 330, 232

Narayan, R., \& Yi, I. 1994, ApJ, 428, L13

Noyola, E., Gebhardt, K., \& Bergmann, M. 2008, ApJ, 676, 1008

Pasquato, M. 2010, arXiv:1008.4477

Pellegrini, S. 2005, ApJ, 624, 155

Pellegrini, S. 2010, ApJ, 717, 640

Peterson, B. M., et al. 2005, ApJ, 632, 799 
Pooley, D., \& Rappaport, S. 2006, ApJ, 644, L45

Portegies Zwart, S. F., Baumgardt, H., Hut, P., Makino, J., \& McMillan, S. L. W. 2004, Nature, 428, 724

Portegies Zwart, S. F., \& McMillan, S. L. W. 2002, ApJ, 576, 899

Quataert, E. 2004, ApJ, 613, 322

Quataert, E., Narayan, R., \& Reid, M. J. 1999, ApJ, 517, L101

Schödel, R., Ott, T., Genzel, R., Eckart, A., Mouawad, N., \& Alexander, T. 2003, ApJ, 596, 1015

Seth, A., Agüeros, M., Lee, D., \& Basu-Zych, A. 2008, ApJ, 678, 116

Shen, S., Mo, H. J., White, S. D. M., Blanton, M. R., Kauffmann, G., Voges, W., Brinkmann, J., \& Csabai, I. 2003, MNRAS, 343, 978

Soria, R., Fabbiano, G., Graham, A. W., Baldi, A., Elvis, M., Jerjen, H., Pellegrini, S., \& Siemiginowska, A. 2006a, ApJ, 640, 126

Soria, R., Graham, A. W., Fabbiano, G., Baldi, A., Elvis, M., Jerjen, H., Pellegrini, S., \& Siemiginowska, A. 2006b, ApJ, 640, 143

Tremaine, S., et al. 2002, ApJ, 574, 740
Trenti, M., Ardi, E., Mineshige, S., \& Hut, P. 2007, MNRAS, 374, 857

Tundo, E., Bernardi, M., Hyde, J. B., Sheth, R. K., \& Pizzella, A. 2007, ApJ, 663,53

Ulvestad, J. S., Greene, J. E., \& Ho, L. C. 2007, ApJ, 661, L151

Umbreit, S., Fregeau, J. M., \& Rasio, F. A. 2009, arXiv:0910.5293

van der Marel, R. P., \& Anderson, J. 2010, ApJ, 710, 1063

van Wassenhove, S., Volonteri, M., Walker, M. G., \& Gair, J. R. 2010, MNRAS, 408, 1139

Volonteri, M. 2007, ApJ, 663, L5

Volonteri, M., Lodato, G., \& Natarajan, P. 2008, MNRAS, 383, 1079

Volonteri, M., \& Natarajan, P. 2009, MNRAS, 400, 1911

Walker, M. G., Mateo, M., Olszewski, E. W., Peñarrubia, J., Wyn Evans, N., \& Gilmore, G. 2009, ApJ, 704, 1274

Walker, M. G., Mateo, M., Olszewski, E. W., Peñarrubia, J., Wyn Evans, N., \& Gilmore, G. 2010, ApJ, 710, 886 